\title{
SEAS UNDER ICE: STABILITY OF LIQUID-WATER OCEANS WITHIN ICY WORLDS.
}

Alberto G. Fairén ${ }^{1,2}$, Javier Ruiz ${ }^{3}$ ${ }^{1}$ Centro de Biología Molecular, Universidad Autónoma de Madrid, 28049, Cantoblanco, Madrid, Spain. (agfairen@cbm.uam.es) ${ }^{2}$ Seminar on Planetary Sciences, Universidad Complutense de Madrid, 28040, Madrid, Spain. ${ }^{3}$ Departamento de Geodinámica, Facultad de Ciencias Geológicas, Universidad Complutense de Madrid, 28040, Madrid, Spain(jaruiz@geo.ucm.es).

Introduction: The possible existence of internal oceans in some icy bodies of the outer Solar System has been suggested through the last years in a joint of observations that cover a wide range of evidences: magnetic fields in Europa and Callisto [1,2], and possibly Ganymede [3], induced by Jupiter's plasma environment in an electricity-conducting layer near the surface of these satellites, likely salty water; hydrated minerals on the surface of Europa [4] and Ganymede [5], which suggest the presence of water on the surface in any moment in the past, with probably an internal source; geological evidences of a mobile layer a few kilometres beneath the surface of Europa [6]; or recent resurfacing in Triton, suggested by its low craterization density [7].

The nature of the different proposed liquid layers could be very different, although the own existence of internal oceans could be a common phenomena. In fact, the conditions invoked to enable the stability of seas under ice layers differ substantially in each world: they come from tidal heating in Europa $[8,9]$ to the stability against convection of the outer ice layer in large satellites as Callisto [10], including the addition of substances as ammonia or salts that could greatly low the melting point of ice $[8,11-14]$ in the Galilean satellites, Titan, Triton, Enceladus or even Pluto.

Here we consider some factors that could affect the thermal state of ice worlds, in order to show that in different (and even opposite) conditions they could hold internal, liquidwater, oceans.

Stress-dependent ice viscosity: Traditionally, it was thought that an ice shell floating over an ocean in a large ice satellite would be unstable against solid-state convection [8], which must drive to the freezing of any liquid water layer in a time no longer than some hundreds of millions of years. An analysis for the case of Callisto [10], taking into account non-Newtonian (stress dependent) viscosity, showed that its outer ice shell should be stable against convection. This implies that the heat provided by radioactive isotopes in the rocky fraction must be enough to let an internal ocean to escape from freezing and survive to date. The argument can be extended to the rest of the icy worlds of the outer Solar System.

Here we have applied the methodology in Ruiz [10] to Titan, using the satellite characteristics as given in Morrison et al. [15]. Deformation mechanisms are indicated as SF (superplastic flow) and DC (dislocation creep), and rheological properties are given in [16,17]. Note (in Figure 1) that Rayleigh number (defined at the base layer, $\mathrm{Ra}_{\mathrm{b}}$ ) in the outer ice shell does not reach a value high enough to initiate convection $\left(\mathrm{Ra}_{\mathrm{b} *}\right.$ is the critical Rayleigh number for the onset of convection, and $b$ is the outer ice shell thickness). So, a water ocean might have survived to date, escaping the deep freezing, at a depth of $\sim 130 \mathrm{~km}$ (deduced from radioactive heat sources power).

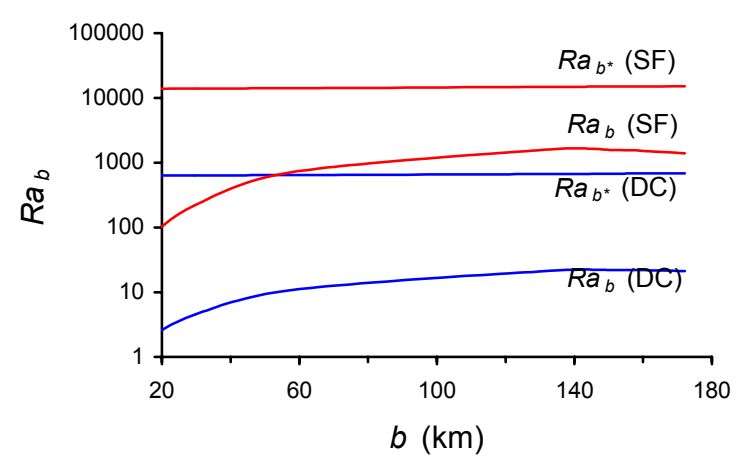

Fig. 1

Possible tidal straining: McKinnon [9] has argued that tidal stresses can reduce ice viscosity, which may allow convection to initiate in the outer shell of Europa. Ganymede may have experimented tidal straining epochs in the past [18], and so tidal stresses could have allowed the onset of convection. We have analysed (using the methods in ref. 19) the stability against convection of an outer shell on Ganymede as a function of surface heat flow, and taking into account a possible range of tidally induced strain rates. An upper limit is given by the tidal strain rates on Europa, estimated in $\sim 10^{-10} \mathrm{~s}^{-1}[20]$, and $10^{-15} \mathrm{~s}^{-1}$ is a strain rate considered typical in many geological processes, both in Earth and other planetary bodies. The results are shown in Figure 2 .

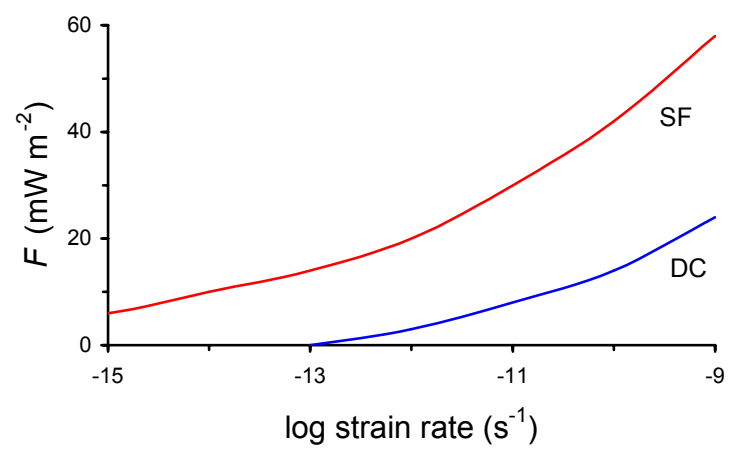

Fig. 2

For the onset of convection, heat flow must descend below the critical value indicated by the red curve for SF, and below the blue curve for DC. So, high strain rates favour the onset of convection. But, in any case, using the approximation in Ruiz and Tejero [19], we have calculated that, for a strain rate of $10^{-10} \mathrm{~s}^{-1}$, tidal heating in the outer shell of Ganymede can stabilise an ice shell $\sim 20-90 \mathrm{~km}$ thick. So, 
although convection could initiate, complete freezing of the internal ocean does not occur.

Spherical geometry on small-size bodies: In previous works about icy worlds, the outer ice shell is frequently treated as a horizontal, "flat" layer. But in the case of smallsize satellites, as Enceladus is, the effect of the real spherical geometry can not be forgotten.

The young appearance of the surface of Enceladus indicates a thermally evolved body [15], which suggests a differentiated interior, maybe due to an ancient tidal heating epoch. In fact, tidal heating calculations in Enceladus including an internal liquid layer indicates that a high heat flow could be possible [21]. Blue curve in Figure 3 shows temperature profile in terms of outer ice layer depth, assuming a cartesian geometry for this layer, temperature-dependent thermal conductivity [22], and Enceladus as tidally heated as suggested. The outer ice shell can be considered heated from below, since warmest the ice is, more tidally heated [20]. Ice melting temperature is reached at $\sim 140 \mathrm{~km}$ depth.

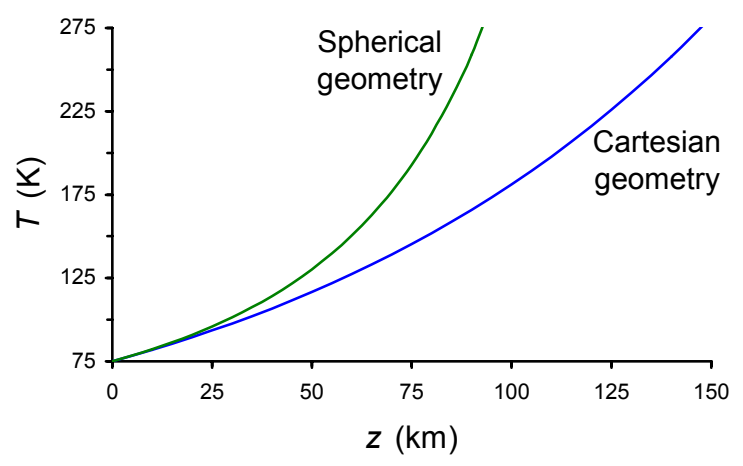

Fig. 3

Otherwise, in a spherical shell in thermal conductive equilibrium, vertical heat flow to a distance $r$ from the center is proportional to $r^{2} / R^{2}=(1-z / R)^{2}$, where $R$ is the body radius and $z$ the depth from surface. When this is taken into account in calculations, the temperature profile represented by the green line is obtained. In this case, melting temperature of water is reached at $\sim 90 \mathrm{~km}$ depth, which greatly increases the possibility of a liquid water layer.

Surface insulating layers: If it exists a regolith layer over the surface of an ice world [23], or if a solid-state greenhouse is working, then temperature can be greatly elevated very close to surface [24]. This could contribute to maintain internal heat, favouring the existence of an internal ocean. Similarly, an ice layer of low thermal conductivity (e.g., nitrogen) over Triton's surface could act as a thermal insulating [11]; this argument can be also applied to Pluto.

The effect of an insulating layer raising surface effective temperature is shown for Triton in Figure 4. It is considered heat transmission by conduction in the ice layer, and radioactive heating in the rocky satellite fraction as the only energy source.

It can be seen that if effective surface temperature is close to observed surface temperature $(38 \mathrm{~K})$, fusion point of water ice (assumed as $273 \mathrm{~K}$ ) is reached at depths up to $\sim 330$ $\mathrm{km}$, similar to those estimated if all water in Triton is frozen. But a more elevated effective surface temperature allows to reach water fusion point at depths close to $\sim 200 \mathrm{~km}$. This would make possible the existence of a water ocean.

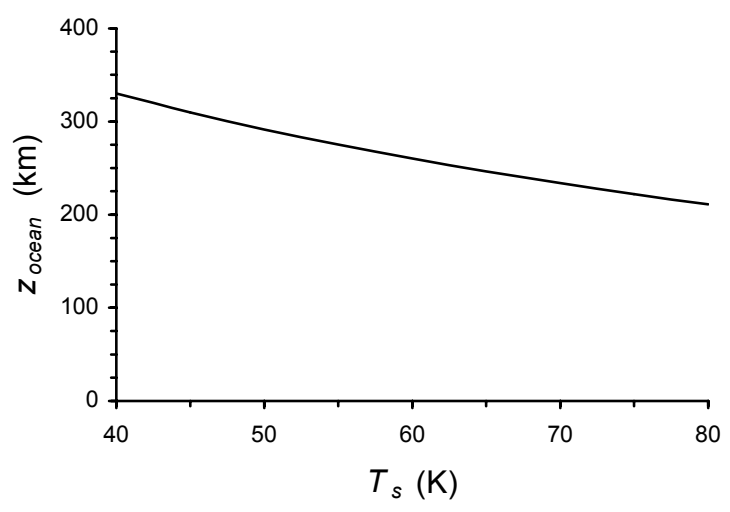

Fig. 4

Conclusions: The existence of liquid water oceans within icy worlds can be a consequence of the physical properties of water ice; and so, addition of antifreeze substances or any other special conditions would not be required. In fact, it is possible that the internal dynamics of these bodies could be very different than usually considered. In any case, the incorporation of significant quantities of substances as ammonia or salts seems to make inevitable $[12,14]$ the existence of different and varied seas under ice in the outer Solar System.

References: [1] Khurana, K. K. et al. (1998) Nature 395, 777780. [2] Zimmer, C. et al. (2000) Icarus 147, 329-347. [3] Kivelson, M G. et al. (2002) Icarus 157, 507-522. [4] McCord, T. B. et al. (1998) Science 280, 1242-1245. [5] McCord, T. B. et al. (2001) Science 292, 1523-1525. [6] Carr M. H. et al. (1998) Nature 391, 363-365. [7] Stern, S. A. and McKinnon, W.B. (1999). LPSC XXX, \#1766. [8] Cassen, P. M. et al. (1982) in Satellites of Jupiter (D. Morrison, Ed.) pp. 93-128. Univ. Ariz. Press, Tucson. [9] McKinnon, W. B. (1999) GRL 26, 951-954. [10] Ruiz, J. (2001) Nature 412, 409-411. [11] McKinnon, W.B. et al. (1995) in Neptune and Triton (D.P. Morrison, Ed.) pp. 807-877, Univ. Ariz. Press, Tucson. [12] Stevenson, D. J. (1998). Eos Trans. AGU Fall Meeting. [13] Kargel, J. S. et al. (2000). Icarus 148, 226-265. [14] Stevenson, D. J. (2001). Eos Trans. AGU Fall Meeting. [15] Morrison, D. et al. (1986) in Satellites (J. Burns and M. S. Matthews, Eds.) pp. 764-801, Univ. Ariz. Press, Tucson. [16] Durham, W. B., et al. (1997) JGR 106, 16,293-16,302. [17] Goldsby, D. L., and D. L. Kohlstedt (2001) JGR 106, 11,017-11,030. [18] Showman, A. P. and R. Malhotra (1997) Icarus 127, 93-111. [19] Ruiz, J. and R. Tejero (2002) Icarus in press. [20] Ojakangas, G. W. and D. J. Stevenson (1989) Icarus 81, 220-241. [21] Ross, M. N., and G. Schubert (1989) Icarus 78, 90-101, 1989. [22] Klinger, J. (1980) Science 209, 271-272. [23] Shoemaker, E. M. et al. (1982) in Satellites of Jupiter (D. Morrison, Ed.) pp. 435-520. Univ. Ariz. Press, Tucson. [24] Matson, D. L. and R. H. Brown (1989) Icarus 77, 67-81. 